\section{Digital Imaging in the}

Materials Engineering Laboratory

Theodore M. Clarke, Case Corporation

The recent completion of a company-wide PC network system, supported by network servers intended to rapidly handle large data files from $\mathrm{CAD}$ systems, made implementation of digital imaging in the Corporate Materials Engineering laboratory of Case Corporation a wise investment. Laboratory reports in Microsoft Word with linked image files can be rapidly received through any of the networked PC's and are archived in the server system. The changeover from film imaging to digital imaging was very sudden. This change was accelerated by satisfied users, whose work was made easier and more productive, and satisfied customers within Case Corporation.

The change to digital imaging occurred with the nearly simultaneous startup of three digital imaging systems in the laboratory. These systems include a JEOL 5800 LV digital SEM with a Noran Voyager EDX system, a video camera connected to a frame grabber for color imaging, and an Eastman Kodak MegaPlus camera, model 1.6i/AB, for high resolution grayscale imaging.

The major investment was in the JEOL 5800 with the Noran Voyager EDX system. Digital images from the SEM are $1280 \times 960$ pixels and can be transferred easily to the Voyager system. The Sun work station on the Voyager is connected to the Token ring network to which the other systems are also connected. The image analysis software on the work station is also used to analyze images from the network connected video camera system and the Kodak system; therefore avoiding additional cost of a dedicated image analysis system for the light microscope. The Kodak MegaPlus camera purchase was influenced by theoretical considerations indicating that it should be capable of nearly matching the resolution and field size of black and white $4 \times 5$ Polaroid prints, our standard for imaging in the materials laboratory.

About half of the images used in Case material evaluation reports are photomacrographs, showing details of machine components. Photomicrographs of microstructures from light microscopes are more frequently used than SEM images in our laboratory. The SEM images are usually fractographs. Nearly all of the laboratory imaging is grayscale. Sometimes color is important, typically when corrosion products with characteristic colors require documentation.

A Javelin video camera was salvaged from an obsolete Photophone system and connected to a ComputerEyes/1024 frame grabber installed in a network connected PC for images requiring color. The images are captured in 256 colors, because $800 \times 600$ resolution in 256 colors is the limit of the PC graphics cards and monitors on the company network. Hard copy is made on network connected 300-dpi color laser printers. VGA output from the camera is used for best image quality rather than composite video output. The ComputerEyes software makes it easy to adjust the hue of the captured image to match the object. The images are normally computer enhanced, which takes only seconds with the ComputerEyes software. The color matching and image resolution appear to be superior to the Polaroid MicroCam, so it is kept as a backup for the digital system. Polaroid $4 \times 5$ color film is available in the laboratory for those wanting higher color image quality. This digital system can also capture grayscale images.

The system using the $1.6 \mathrm{i}$ Kodak camera, model $1.6 \mathrm{i} / \mathrm{AB}$, is now the predominant imaging system used in the laboratory for grayscale imaging. The camera has an antiblooming sensor, which is an extra cost feature but absolutely necessary for imaging part surfaces with unavoidable specular reflections of the light sources. Image resolution is also slightly improved with the antiblooming sensor. The camera has a $9 \times 13 \mathrm{~mm}$ CCD sensor with $1534 \times 1024$ pixel elements and the digital output of the camera is 10-bit grayscale. The Kodak camera is connected to a Digital Equipment Corp. 90-megahertz Pentium PC with 40 megabytes of RAM, a Coreco Oculusf/64 image acquisition system and graphics card. The system has an NEC XP21 $1600 \times 1200$ pixel monitor. The 1.2-gigabyte hard drive has Image Central software from Advanced Imaging Concepts so that an image database system can be built with reference images and associated data.
The cost of this system is less than that of a modern research metallograph, which would have no better resolution than the 1970's vintage Zeiss Universal Microscope on which the camera is mounted. This microscope has metaliurgical objectives ranging from $2.5 \times(0.08$ N.A.) to $100 \times(1.25$ N.A.). A Diagnostics Instruments adapter, designed to be compatible with the flat field compensating eyepiece Zeiss system, images the same field size on the Kodak CCD sensor as would normally be recorded on $4 \times 5$ film. The Kodak camera has a Nikon "F" mount so that it can be used with 35- mm camera lenses on a Kaiser copy stand with the PC and monitor between the microscope and copy stand. Camera transfer from microscope use to copy stand use takes less than a minute. The Kodak camera has an electromagnetic actuated shutter which is controlled through a menu on the $1600 \times 1200$ pixel 21 inch monitor. Camera display is set at $1280 \times 1024$ pixels to avoid the use of dual monitors, a limitation which future systems should not have.

Maximum resolution is desired and exposure times are less than 1 second, so the gain and black level default values are set at zero. This is important for users who might forget to check these settings and obtain inferior image quality. Focusing of images from the microscope and setting the shutter speed are very easily accomplished while viewing the monitor. Monitor brightness is preset based upon getting a match in grayscale detail between the monitor and laser prints. The image acquisition software is the demonstration program provided with the f/64 system. The next revision of this software will have file overwrite protection along with pixel gray level histogram plotting. Computer enhancement (high pass filter) is normally used to sharpen all of the images, and is very quick with the f/64 system. All of the image files to be used in reports are coded with a job number obtained from Borland Paradox database system used for tracking all work in the laboratory. Image file code includes the consecutive image number and letters for the standardized image recording conditions. The images are filed on a network server and a laser print is immediately made on a 600-dpi network printer set so the 1280 pixel width is eight inches wide on the hardcopy. Laser print resolution is almost as good as the monitor image, which is normally scrolled in a $640 \times 480$ pixel window on monitors set for $800 \times 600$ resolution. The contrast range of the laser prints is much lower than the 256 gray levels of the monitor. This is a more important deficiency than the loss of resolution in the laser prints. Adobe Photoshop can be used to increase the contrast for printing, but this may cause an objectionable loss of highlight and shadow detail. Notation is added below the initial prints for future reference. Scale bars can be added by the materials engineer when he views and marks up an image for a final report, using Paintbrush or Adobe Photoshop. Images are stored as 1.31-megabyte "bmp" files rather than "tif" files, because all of the networked PC's have Windows Paintbrush for viewing the images. "Tif" file sizes and image quality are the same as the "bmp" files.

As previously noted, about half of all images used in the material evaluation report are photomacrographs. It is in this application that the Kodak MegaPlus camera system has shown the greatest advantage over film imaging in our laboratory. The photomacrographs are typically of fracture surfaces and relevant features of a machine component. These are not flat surfaces and are often, in the case of gear tooth flanks or bearing components, spectrally reflecting. Establishing lighting conditions and the right camera exposure to show the desired details is much more difficult than photomicroscopy. Obtaining adequate depth of field is a further complicating factor. The digital system allows rapid establishment of illumination, focus, and depth of field while viewing the monitor. No time and film is wasted on test exposures. Time savings average at least $75 \%$. The lenses used for photomacrography on the camera stand are a $28-\mathrm{mm} f / 2.8$ Vivitar, 60-mm f/2 Nikon Micro Nikkor, a 100-mm f/6.3 Zeiss Luminar, and a 63 $\mathrm{mm} \mathrm{f} / 4.5$ Zeiss Luminar. These lenses were designed to cover a $35-\mathrm{mm}$ film format of $24 \times 36 \mathrm{~mm}$, but the Kodak camera's CCD sensor records only a $9 \times 13 \mathrm{~mm}$ - portion of the image formed by the lens. The digital images are equivalent to $4 \times 5$ Polaroid prints of the same fields with a maximum print resolution of about 5.6 lines $/ \mathrm{mm}$, requiring a lens resolution of about 60 lines $/ \mathrm{mm}$ at the CCD sensor. The aperture setting to just achieve this resolution can be calculated from this equation:

$$
\text { f/mumber }=24 /(\text { Camera Mag. }+1)
$$

This calculation assumes the digital image is equivalent to a $10 \mathrm{X}$ enlargement of a portion of the image formed by the lens on the sensor to a $3.5 \times 4.5$ print size. A digital image resulting from the $60 \mathrm{~mm}$ Nikon lens focused at $1 X$ camera 
magnification, with a lens aperture setting of $f / 11$, is equivalent to a good quality $10 X$ photomacrograph recorded on $4 \times 5$ Polaroid film. Zeiss Luminar lenses are used for higher magnifications. The $100-\mathrm{mm}$ Luminar lens, optimized for $2 X$ camera magnification, is used to cover field sizes equivalent to $4 \times 5$ Polaroid prints with magnifications between $10 \mathrm{X}$ and $25 \mathrm{X}$. A $63-\mathrm{mm}$ Luminar covers a field size range equivalent to $25 \mathrm{X}-50 \mathrm{X}$ Polaroids. An extension tube was made in my home machine shop to connect the Zeiss lenses to the bayonet mount of an Olympus Auto Bellows. The Olympus Auto Bellows camera connection ring was modified to Nikon "F" mount to connect the Kodak MegaPlus camera to the bellows. This system provides an advantage of long working distance, $2 \frac{1}{4}$ " for $50 \mathrm{X}$ and $4^{\prime \prime}$ for $25 \mathrm{X}$. Since camera height adjustment on the copy stand is not sensitive enough for easy focusing at the higher magnifications, samples are placed on a fine focusing stage if their size and weight permit. A future improvement will be to modify the vertical illuminator for the $100-\mathrm{mm}$ Luminar on the Ultraphot II so it can be used with the $60-\mathrm{mm}$ Nikon lens and the $100-\mathrm{mm}$ Luminar on the digital imaging system. Initial testing indicates that this illuminator will then cover a much larger field size than when used on the Ultraphot II. This will allow the digital camera to replace Polaroid film currently used for this application and the time spent digitizing these film images.

It is hoped this review of the introduction of high resolution digital imaging in the Case Corporation Materlals Engineering Laboratory is helpful for other laboratories considering the changeover to digital imaging. However, the current cost of this technology makes $4 \times 5$ Polaroid and $35-\mathrm{mm}$ film imaging the most cost effective means of imaging for smaller laboratories and the film images are superior to those from all but the most expensive digital cameras. This situation will rapidly change as high resolution CCD camera system costs drop. CCD arrays of $3000 \times 2000$ and then $6000 \times 4000$ will become affordable and eliminate the current advantage of film resolution. General user image acquisition systems should become available soon so that scale bars can be added at the time the image is acquired, perhaps automatically with computer links to the light microscope and macro imaging system. High resolution zoom lenses with brightfield illumination attachments will replace the bellows lenses. Aperture settings for the microscope illumination and the macro zoom lenses could be automatically set to values that provide an optimum combination of resolution and depth of field. Concurrent with these improvements will be improved ability to transmit, store, view, and print the digital images at full resolution in the sizes used for high quality photographs.

We will be happy to answer any questions raised by this article. An enhanced version of this article, with digital images and photographs of the system, will be submitted for publication in "The Microscope".

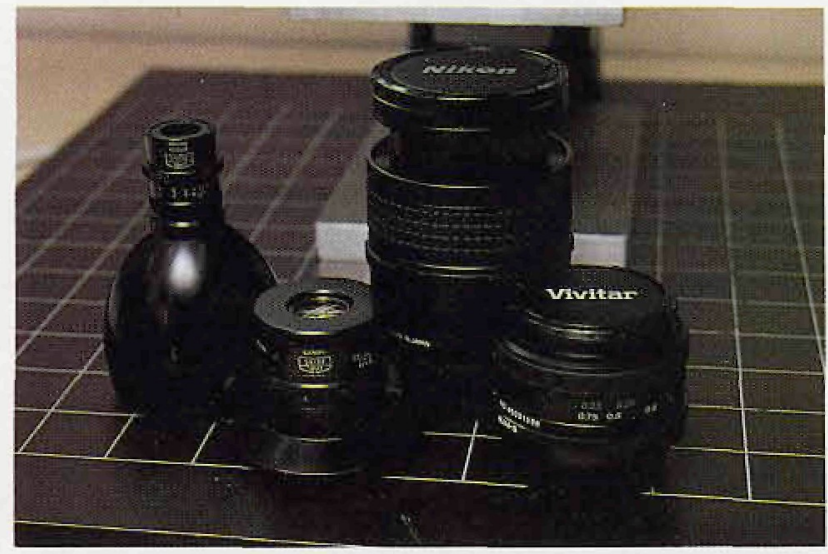

Four lenses are used for photomacography using the Kodak MegaPlus camera, model 1.6i/AB. They are (from left) a $63 \mathrm{~mm}$ Zeiss Luminar lens (for magnifications from 25-50X, in combination with the Olympus bellows system); a $100 \mathrm{~mm}$ Zeiss Luminar lens (for magnifications from 10-25, in combination with the Olympus bellows system); a $60 \mathrm{~mm}$ Micro Nikkor lens (for magnifications from $1 \mathrm{X}$ to $10 \mathrm{X})$; and a $28 \mathrm{~mm} \mathrm{f} / 2.8$ Vivitar lens for large field images/

Acknowledgement

The successful transition to digital imaging in the Corporate Materials
Engineering laboratory of Case Corporation was a team effort. Help from materials engineers Dave Dobbins and Ed Rahe with the computer system and the method for transmitting the reports, where image files linked to a server rather than saved in the document, is gratefully acknowledged.

Reference

1. Clarke,T.M., "Light Microscopy Criteria for Electronic Imaging"; MICROSCOPE $1995,43: 3,121-124$

Reprinted with permission from $\mu$ - NOTES 2000, a State Microscopical Society of Illinois publication. To join this Society and receive their most interesting newsletter, contact Bill Mikuska, $\mu$ - NOTES $2000,2820 \mathrm{~S}$. Michigan Ave, Chicago, IL 60616, Tel.: (312)842-7100.

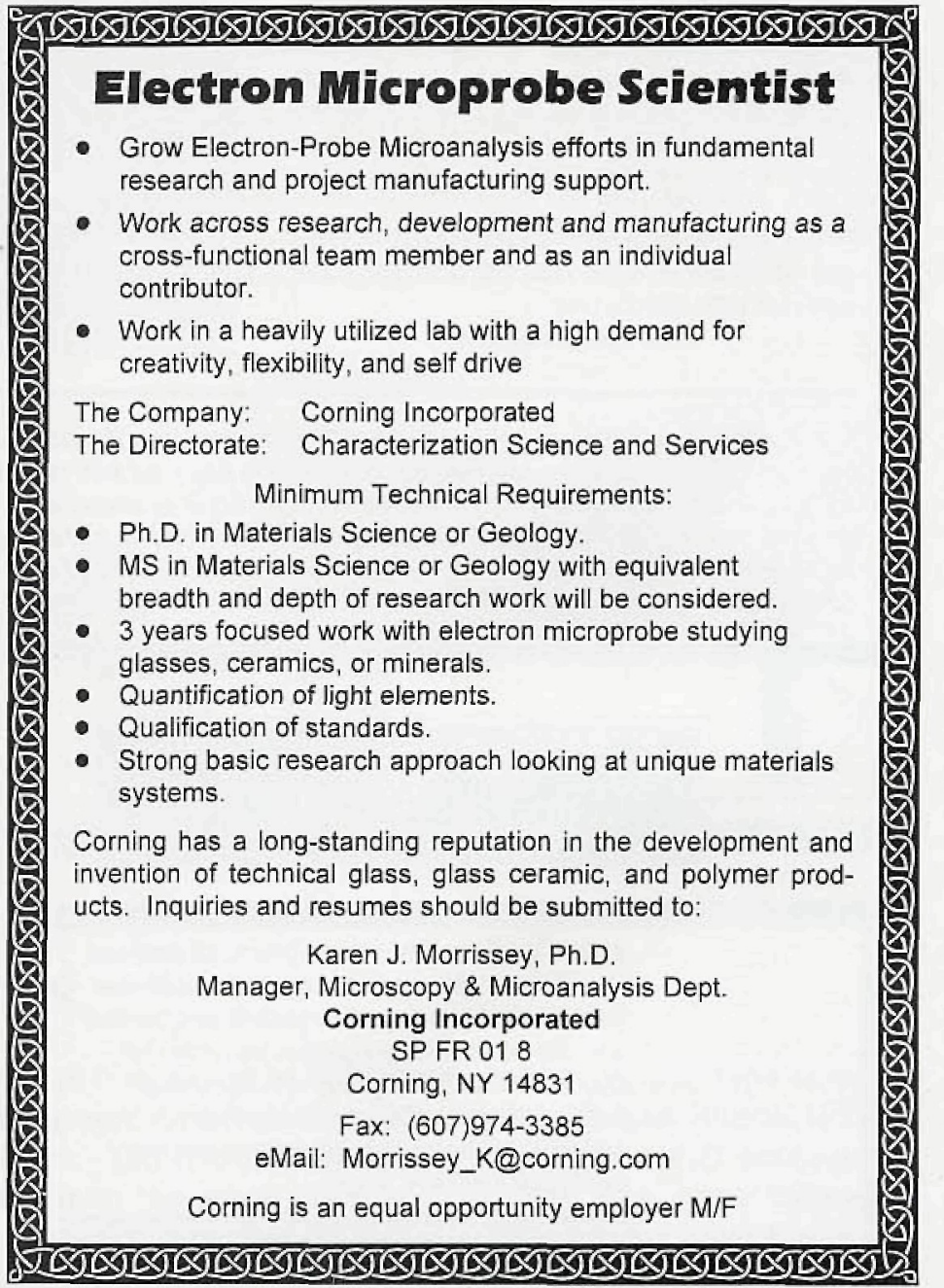

Call to request our new catalog! Quality E.M. supplies
"taylored" to fit your
application and budget!

M.E. TAYLOR ENGINEERING, INC.

21604 Gentry Lane • Brookeville, MD 20833

Phone: 1-301-774-6246 - FAX: 1-301-774-6711

E-Mail: Metengr@aol.com

VISA AND MASTERCARD ACCEPTED! 